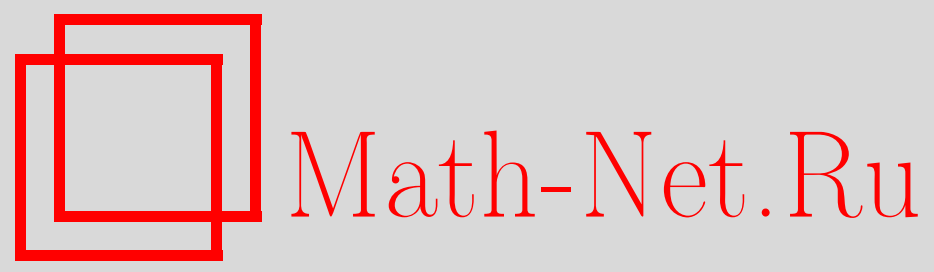

С. А. Степанов, Новый класс нелинейных пятеричных кодов, Дискрет. матем., 2005, том 17, выпуск 4, 7-17

DOI: https://doi.org/10.4213/dm125

Использование Общероссийского математического портала Math-Net.Ru подразумевает, что вы прочитали и согласны с пользовательским соглашением http://www.mathnet.ru/rus/agreement

Параметры загрузки:

IP : 54.237 .206 .68

26 апреля 2023 г., 08:53:03 


\title{
Новый класс нелинейных пятеричных кодов
}

\author{
() 2005 г. . А. Степанов
}

\begin{abstract}
В статье построены два новых класса нелинейных пятеричных кодов, получаемых из соответствующих классов модифицированных матриц Батсона-Адамара. Эти коды имеют минимальное расстояние, близкое к границе Плоткина, а также простые процедуры построения и декодирования.

Работа выполнена при поддержке Института экспертизы информационных технологий, Южная Корея.
\end{abstract}

\section{1. Введение}

Многие преобразователи дискретных сигналов основаны на использовании преобразований матриц с элементами, принимающими значения на единичной окружности в комплексной плоскости, например, семейство обобщенных дискретных преобразователей сигналов длины $n=2^{m}$ (см. раздел 10.2 в [4]). Это семейство включает в себя преобразование Уолша-Адамара и $2^{m}$-точечное дискретное преобразование Фурье. Интерпретация быстрого Куули-Турки преобразования Фурье в терминах характеров абелевой группы (см., например, [6]) показывает, что дискретное преобразование Фурье само является обобщенным преобразованием, включающим преобразование Уолша-Адамара.

Все матрицы упоминаемых выше преобразований содержатся в классе матриц Батсона-Адамара [3], являющемся в свою очередь подклассом коциклических обобщенных матриц Адамара. Обобщенные матрицы Адамара и коциклические обобщенные матрицы Адамара использовались различными авторами (см. $[1,2,5,6])$ для построения соответствующих кодов Адамара и кощиклических кодов Адамара.

В этой работе мы вводим два новых класса $\left\{H_{m}\right\},\left\{\widetilde{H}_{m}\right\}$ модифицированных матриц Батсона-Адамара $H_{m}, \widetilde{H}_{m}$ с элементами из $C=\left\{1, \omega, \omega^{2}, \omega^{3}, \omega^{4}, \omega^{5}\right\}$, где $\omega-$ примитивный комплексный корень пятой степени из единицы, и строим два класса $\left\{C_{m}^{*}\right\},\left\{\widetilde{C}_{m}^{*}\right\}$ нелинейных пятеричных кодов $C_{m}^{*}, \widetilde{C}_{m}^{*}$, получаемых соответственно из $H_{m}$ и $\widetilde{H}_{m}$. Главная цель статьи состоит в доказательстве следующей теоремы.

Теорема 1. Коды $C_{m}^{*} u \widetilde{C}_{m}^{*}$ имеют параметры

$$
\left(3^{m}, 5^{m}, 2 \cdot 3^{m-1}\right), \quad\left(3^{m}, 5^{m+1}, 2 \cdot 3^{m-1}\right)
$$

и исправляют любые конфигурачии из $t \leqslant\left[\left(2 \cdot 3^{m-1}-1\right) / 2\right]$ ошибок.

Теорема 1 показывает, что минимальное расстояние кодов $C_{m}^{*}$ и $\tilde{C}_{m}^{*}$ близко к гранище Плоткина. 


\section{2. Модифицированные матрицы Батсона-Адамара}

Пусть $\omega=e^{2 \pi i / 5}-$ примитивный корень пятой степени из единицы и $\mathbf{Q}(\omega)$ - поле деления круга, получаемое из поля рациональных чисел $\mathbf{Q}$ присоединением $\omega$. Минимальный многочлен над полем $\mathbf{Q}$ имеет вид

$$
f(x)=1+x+x^{2}+x^{3}+x^{4} .
$$

Элементы $1, \omega, \omega^{2}, \omega^{3}$ образуют базис поля $\mathbf{Q}(\omega)$ над $\mathbf{Q}$, поэтому любое $\alpha \in \mathbf{Q}(\omega)$ единственным образом представляется в виде линейной комбинации

$$
\alpha=a_{0}+a_{1} \omega+a_{2} \omega^{2}+a_{3} \omega^{3}
$$

базисных элементов с коэффищиентами $a_{0}, a_{1}, a_{2}, a_{3} \in \mathbf{Q}$.

Пусть $\mathbf{Z}$ - поле рациональных целых чисел. Мы работаем в кольце $\mathbf{Z}[\omega]$ алгебраических целых чисел поля $\mathbf{Q}(\omega)$. Каждый элемент $\alpha \in \mathbf{Z}[\omega]$ единственным образом представляется в виде

$$
\alpha=a_{0}+a_{1} \omega+a_{2} \omega^{2}+a_{3} \omega^{3},
$$

где $a_{0}, a_{1}, a_{2}, a_{3} \in \mathbf{Z}$. Кольцо алгебраических целых чисел $\mathbf{Z}[\omega]$ содержит мультипликативную циклическую группу $C=\langle\omega\rangle=\left\{1, \omega, \omega^{2}, \omega^{3}, \omega^{4}, \omega^{5}\right\}$ порядка 5 с элементами, удовлетворяюшими соотношению

$$
1+\omega+\omega^{2}+\omega^{3}+\omega^{4}=0 .
$$

Рассмотрим матрицу Батсона-Адамара

$$
\left(\begin{array}{ccccc}
1 & 1 & 1 & 1 & 1 \\
1 & \omega & \omega^{2} & \omega^{3} & \omega^{4} \\
1 & \omega^{2} & \omega^{4} & \omega & \omega^{3} \\
1 & \omega^{3} & \omega & \omega^{4} & \omega^{2} \\
1 & \omega^{4} & \omega^{3} & \omega^{2} & \omega
\end{array}\right)
$$

и составим из элементов этой матрищы матрицу

$$
H_{1}=\left(\begin{array}{ccc}
1 & 1 & 1 \\
1 & \omega & \omega^{2} \\
1 & \omega^{2} & \omega^{4} \\
1 & \omega^{3} & \omega \\
1 & \omega^{4} & \omega^{3}
\end{array}\right)
$$

Пусть $A \otimes B$ обозначает кронекерово произведение матриц $A$ и $B$. Определим модифицированные матрищы Батсона-Адамара $H_{m}$ и $\widetilde{H}_{m}$ для $m=2,3, \ldots$, рекурсивно с помощью соотношений

$$
H_{m}=H_{1} \otimes H_{m-1}=\left(\begin{array}{ccc}
H_{m-1} & H_{m-1} & H_{m-1} \\
H_{m-1} & \omega H_{m-1} & \omega^{2} H_{m-1} \\
H_{m-1} & \omega^{2} H_{m-1} & \omega^{4} H_{m-1} \\
H_{m-1} & \omega^{3} H_{m-1} & \omega H_{m-1} \\
H_{m-1} & \omega^{4} H_{m-1} & \omega^{3} H_{m-1}
\end{array}\right)
$$


и

$$
\tilde{H}_{m}=\left(\begin{array}{c}
H_{m} \\
\omega H_{m} \\
\omega^{2} H_{m} \\
\omega^{3} H_{m} \\
\omega^{4} H_{m}
\end{array}\right) .
$$

Ясно, что $H_{m}$ и $\tilde{H}_{m}$ представляют собой соответственно $5^{m} \times 3^{m}$ и $5^{m+1} \times 3^{m}$ матрищы, составленный из элементов циклической группы $C=\langle\omega\rangle$. Пусть

$$
H_{1}^{*}=\left(\begin{array}{ccccc}
1 & 1 & 1 & 1 & 1 \\
1 & \omega^{4} & \omega^{3} & \omega^{2} & \omega \\
1 & \omega^{3} & \omega & \omega^{4} & \omega^{2}
\end{array}\right)
$$

- матрица, эрмитово сопряженная с матрицей $H_{1}$. Если $H_{m-1}^{*}$ - эрмитово сопряженная матрища с матрищей $H_{m-1}$, то эрмитово сопряженная матрища с матрищей $H_{m}$ равна

$$
H_{m}^{*}=\left(\begin{array}{ccccc}
H_{m-1}^{*} & H_{m-1}^{*} & H_{m-1}^{*} & H_{m-1}^{*} & H_{m-1}^{*} \\
H_{m-1}^{*} & \omega^{4} H_{m-1}^{*} & \omega^{3} H_{m-1}^{*} & \omega^{2} H_{m-1}^{*} & \omega H_{m-1}^{*} \\
H_{m-1}^{*} & \omega^{3} H_{m-1}^{*} & \omega H_{m-1}^{*} & \omega^{4} H_{m-1}^{*} & \omega^{2} H_{m-1}^{*}
\end{array}\right) .
$$

Рассмотрим теперь самосопряженную матрицу

$$
J_{1}=H_{1} H_{1}^{*},
$$

которая имеет вид

$$
\left(\begin{array}{ccccc}
3 & 1+\omega^{3}+\omega^{4} & 1+\omega+\omega^{3} & 1+\omega^{2}+\omega^{4} & 1+\omega+\omega^{2} \\
1+\omega+\omega^{2} & 3 & 1+\omega^{3}+\omega^{4} & 1+\omega+\omega^{3} & 1+\omega^{2}+\omega^{4} \\
1+\omega^{2}+\omega^{4} & 1+\omega+\omega^{2} & 3 & 1+\omega^{3}+\omega^{4} & 1+\omega+\omega^{3} \\
1+\omega+\omega^{3} & 1+\omega^{2}+\omega^{4} & 1+\omega+\omega^{2} & 3 & 1+\omega^{3}+\omega^{4} \\
1+\omega^{3}+\omega^{4} & 1+\omega+\omega^{3} & 1+\omega^{2}+\omega^{4} & 1+\omega+\omega^{2} & 3
\end{array}\right)
$$

и заметим, что все действительные части чисел

$$
1+\omega+\omega^{2}, \quad 1+\omega+\omega^{3}, \quad 1+\omega^{2}+\omega^{4}, \quad 1+\omega^{3}+\omega^{4}
$$

равны $1 / 2$. Наконец, определим комплексно самосопряженные матрищы $J_{m}$ для $m=$ $2,3, \ldots$, с помощью рекуррентного соотношения

$$
J_{m}=J_{1} \otimes J_{m-1}
$$

Так как

$$
H_{1} H_{1}^{*}=J_{1} \text {, }
$$

то для любого $m \geqslant 1$

$$
H_{m} H_{m}^{*}=J_{m}
$$

Два последних соотношения показывают, что матрищы $H_{m}$ и $\tilde{H}_{m}$ не входят в класс обобщенных матрищ Адамара. С другой стороны, используя индукщию по $m$, нетрудно показать, что справедливо следующее утверждение. 
Предложение 1. Для любого $m \geqslant 1$ справедливо равенство $J_{m}^{*}=J_{m}$. Элементы $\gamma_{k l}$, $k, l=1, \ldots, 5^{m}$, матрищы $J_{m}$ являются алгебраическими челыми числами из $\mathbf{Z}[\omega]$ вида

$$
\gamma_{k l}=c_{k l}^{(0)}+c_{k l}^{(1)} \omega+c_{k l}^{(2)} \omega^{2}+c_{k l}^{(3)} \omega^{3},
$$

где $c_{k l}^{(0)}, c_{k l}^{(1)}, c_{k l}^{(2)}, c_{k l}^{(3)} \in \mathbf{Z}$ и все диагональные элементы матричы $J_{m}$ равны $3^{m}$. Действительные части недиагональных элементов $\gamma_{k l}$ не превосходят $3^{m-1} / 2$.

Этот результат и соотношения (2) показывают что матрищы $H_{m}$ обладают рядом свойств, которые во многих аспектах аналогичны основным свойствам классических матриц Адамара.

\section{3. Модифицированные коды Адамара}

Пусть $H_{m}$ и $\tilde{H}_{m}$ - модифицированные матрицы Батсона-Адамара, определенные в предыдущем разделе. Модифищированный пятеричный код Адамара $C_{m}^{*}$ определяется как множество столбцов матрицы $H_{m}^{*}$, эрмитово сопряженной с матрицей $H_{m}$. Аналогично, модифицированный пятеричный код Адамара $\tilde{C}_{m}^{*}$ состоит из столбцов матрицы $\tilde{H}_{m}^{*}$, эрмитово сопряженной с матрищей $\widetilde{H}_{m}$. Ясно, что $C_{m}^{*}$ и $\widetilde{C}_{m}^{*}-$ нелинейные $\left(3^{m}, 5^{m}, d\right)$ и $\left(3^{m}, 5^{m+1}, d\right)$ коды соответственно.

Найдем минимальное расстояния Хемминга $d$ кодов $C_{m}^{*}$ и $\widetilde{C}_{m}^{*}$. Рассмотрим матрицу

$$
H_{m}=\left(\begin{array}{ccc}
H_{m-1} & H_{m-1} & H_{m-1} \\
H_{m-1} & \omega H_{m-1} & \omega^{2} H_{m-1} \\
H_{m-1} & \omega^{2} H_{m-1} & \omega^{4} H_{m-1} \\
H_{m-1} & \omega^{3} H_{m-1} & \omega H_{m-1} \\
H_{m-1} & \omega^{4} H_{m-1} & \omega^{3} H_{m-1}
\end{array}\right)
$$

как упорядоченное множество строк. Следующий результат играет центральную роль в дальнейших рассмотрениях.

Теорема 2. Пусть $\alpha_{k} u \alpha_{l}-$ две строки матрицы $H_{m} u \omega^{r}, \omega^{s}-$ два элемента группы $C=\left\{1, \omega, \omega^{2}, \omega^{3}, \omega^{4}\right\}$. Если $\omega^{r} \alpha_{k} \neq \omega^{r} \alpha_{l}$, то расстояние Хемминга межуду $\omega^{r} \alpha_{k} u \omega^{s} \alpha_{s}$ не меньие $2 \cdot 3^{m-1}$.

Доказательство. Докажем теорему индукщией по $m$. При $m=1$ множество элементов $\omega^{\sigma} \alpha_{k}$, где $\alpha_{k} \in H_{1}$ и $\omega^{\sigma} \in C$, совпадает с множеством строк матрищ

$$
\begin{aligned}
H_{1} & =\left(\begin{array}{ccc}
1 & 1 & 1 \\
1 & \omega & \omega^{2} \\
1 & \omega^{2} & \omega^{4} \\
1 & \omega^{3} & \omega \\
1 & \omega^{4} & \omega^{3}
\end{array}\right), \quad \omega H_{1}=\left(\begin{array}{ccc}
\omega & \omega & \omega \\
\omega & \omega^{2} & \omega^{3} \\
\omega & \omega^{3} & 1 \\
\omega & \omega^{4} & \omega^{2} \\
\omega & 1 & \omega^{4}
\end{array}\right), \quad \omega^{2} H_{1}=\left(\begin{array}{ccc}
\omega^{2} & \omega^{2} & \omega^{2} \\
\omega^{2} & \omega^{3} & \omega^{4} \\
\omega^{2} & \omega^{4} & \omega \\
\omega^{2} & 1 & \omega^{3} \\
\omega^{2} & \omega & 1
\end{array}\right), \\
\omega^{3} H_{1} & =\left(\begin{array}{cccc}
\omega^{3} & \omega^{3} & \omega^{3} \\
\omega^{3} & \omega^{4} & 1 \\
\omega^{3} & 1 & \omega^{2} \\
\omega^{3} & \omega & \omega^{4} \\
\omega^{3} & \omega^{2} & \omega
\end{array}\right), \omega^{4} H_{1}=\left(\begin{array}{ccc}
\omega^{4} & 1 & \omega \\
\omega^{4} & \omega & \omega^{3} \\
\omega^{4} & \omega^{2} & 1 \\
\omega^{4} & \omega^{3} & \omega^{2}
\end{array}\right) .
\end{aligned}
$$


Это показывает, что расстояние Хемминга между любыми двумя различными строками $\omega^{\sigma} \alpha_{k}$ и $\omega^{\tau} \alpha_{l}$ не меньше 2 . Поэтому при $m=1$ теорема верна.

Пусть теперь $m \geqslant 2$. Рассмотрим подматрицы

$$
\begin{aligned}
& H_{m-1}^{(0,0,0)}=\left(\begin{array}{lll}
H_{m-1} & H_{m-1} & H_{m-1}
\end{array}\right) \\
& H_{m-1}^{(0,1,2)}=\left(\begin{array}{lll}
H_{m-1} & \omega H_{m-1} & \omega^{2} H_{m-1}
\end{array}\right) \\
& H_{m-1}^{(0,2,4)}=\left(\begin{array}{lll}
H_{m-1} & \omega^{2} H_{m-1} & \omega^{4} H_{m-1}
\end{array}\right) \\
& H_{m-1}^{(0,3,1)}=\left(\begin{array}{lll}
H_{m-1} & \omega^{3} H_{m-1} & \omega H_{m-1}
\end{array}\right) \\
& H_{m-1}^{(0,4,3)}=\left(\begin{array}{lll}
H_{m-1} & \omega^{4} H_{m-1} & \omega^{3} H_{m-1}
\end{array}\right)
\end{aligned}
$$

матрицы $H_{m}$. Из предыдущего следует, что для матрицы $H_{m}$ единственно возможными являются строки $\alpha_{k}$ вида

$$
\begin{aligned}
\alpha_{k} & =\left(\begin{array}{lll}
a_{k} & a_{k} & a_{k}
\end{array}\right), \\
\alpha_{k} & =\left(\begin{array}{lll}
a_{k} & \omega a_{k} & \omega^{2} a_{k}
\end{array}\right), \\
\alpha_{k} & =\left(\begin{array}{lll}
a_{k} & \omega^{2} a_{k} & \omega^{4} a_{k}
\end{array}\right), \\
\alpha_{k} & =\left(\begin{array}{lll}
a_{k} & \omega^{3} a_{k} & \omega a_{k}
\end{array}\right), \\
\alpha_{k} & =\left(\begin{array}{lll}
a_{k} & \omega^{4} a_{k} & \omega^{3} a_{k}
\end{array}\right),
\end{aligned}
$$

где $a_{k} \in H_{m-1}$.

Предположим, что расстояние Хемминга между двумя различными векторами $\omega^{r} a_{k}$, $\omega^{s} a_{l}$, где $a_{k}, a_{l} \in H_{m-1}, \omega^{r}, \omega^{s} \in C$, не меньше $2 \cdot 3^{m-2}$, и докажем, что расстояние Хемминга между двумя различными векторами $\omega^{\sigma} \alpha_{k}$ и $\omega^{\tau} \alpha_{l}$, где $\alpha_{k}, \alpha_{l} \in H_{m}, \omega^{\sigma}, \omega^{\tau} \in C$, не меньше $2 \cdot 3^{m-1}$. Рассмотрим векторы

$$
\begin{aligned}
\alpha_{k} & =\left(\begin{array}{llll}
a_{k} & \omega^{\delta} a_{k} & \omega^{\lambda} a_{k}
\end{array}\right), & \alpha_{l} & =\left(\begin{array}{llll}
a_{l} & \omega^{\mu} a_{l} & \omega^{\nu} a_{l}
\end{array}\right), \\
\omega^{\sigma} \alpha_{k} & =\left(\begin{array}{lllll}
\omega^{\sigma} a_{k} & \omega^{\sigma+\delta} a_{k} & \omega^{\sigma+\lambda} a_{k}
\end{array}\right), & \omega^{\tau} \alpha_{l} & =\left(\begin{array}{llll}
\omega^{\tau} a_{l} & \omega^{\tau+\mu} a_{l} & \omega^{\tau+\nu} a_{l}
\end{array}\right)
\end{aligned}
$$

и заметим, что расстояние Хемминга между $\omega^{\sigma} a_{k}$ и $\omega^{\tau} a_{l}$ равно

$$
d\left(\omega^{\sigma} \alpha_{k}, \omega^{\tau} \alpha_{l}\right)=d\left(\omega^{\sigma} a_{k}, \omega^{\tau} a_{l}\right)+d\left(\omega^{\sigma+\delta} a_{k}, \omega^{\tau+\mu} a_{l}\right)+d\left(\omega^{\sigma+\lambda} a_{k}, \omega^{\tau+\nu} a_{l}\right) .
$$

Заметим также, что если $\omega^{\sigma} a_{k}=\omega^{\tau} a_{l}$, то $\sigma=\tau$ и $a_{k}=a_{l}$. Действительно, из того, что первые компоненты векторов $a_{k}$ и $a_{l}$ равны 1 , следует, что $\sigma=\tau$, так что $a_{k}=a_{l}$. Для того, чтобы найти нетривиальную нижнюю границу для $d\left(\omega^{\sigma} \alpha_{k}, \omega^{\tau} \alpha_{l}\right)$, рассмотрим следующие четыре случая.

1. Пусть $(\delta, \lambda)=(\mu, v)=(0,0)$. Тогда

$$
d\left(\omega^{\sigma} \alpha_{k}, \omega^{\tau} \alpha_{l}\right)=3 d\left(\omega^{\sigma} a_{k}, \omega^{\tau} a_{l}\right),
$$

поэтому из того, что $d\left(\omega^{\sigma} \alpha_{k}, \omega^{\tau} \alpha_{l}\right) \neq 0$, следует, что $d\left(\omega^{\sigma} a_{k}, \omega^{\tau} a_{l}\right) \neq 0$. Если $l=k$ и $\sigma \neq \tau$, то $d\left(\omega^{\sigma} a_{k}, \omega^{\tau} a_{k}\right)=3^{m-1}$ и поэтому

$$
d\left(\omega^{\sigma} \alpha_{k}, \omega^{\tau} \alpha_{l}\right)=3^{m}>2 \cdot 3^{m-1} .
$$

Если $l \neq k$, то по предположению индукщии

$$
d\left(\omega^{\sigma} a_{k}, \omega^{\tau} a_{l}\right) \geqslant 2 \cdot 3^{m-2},
$$


и следовательно,

$$
d\left(\omega^{\sigma} \alpha_{k}, \omega^{\tau} \alpha_{l}\right) \geqslant 2 \cdot 3^{m-1}
$$

2. Пусть $(\delta, \lambda)=(0,0)$ и $\mu \neq v-$ ненулевой элемент множества $\{0,1,2,3,4\}$. Рассмотрим следующие подслучаи.

2.1. Пусть $\omega^{\sigma} a_{k} \neq \omega^{\tau} a_{l}, \omega^{\sigma} a_{k} \neq \omega^{\tau+\mu} a_{l}$ и $\omega^{\sigma} a_{k} \neq \omega^{\tau+v} a_{l}$. По предположению индукщии

$$
\begin{aligned}
d\left(\omega^{\sigma} a_{k}, \omega^{\tau} a_{l}\right) & \geqslant 2 \cdot 3^{m-2}, \\
d\left(\omega^{\sigma} a_{k}, \omega^{\tau+\mu} a_{l}\right) & \geqslant 2 \cdot 3^{m-2}, \\
d\left(\omega^{\sigma} a_{k}, \omega^{\tau+\nu} a_{l}\right) & \geqslant 2 \cdot 3^{m-2},
\end{aligned}
$$

и следовательно,

$$
d\left(\omega^{\sigma} \alpha_{k}, \omega^{\tau} \alpha_{l}\right) \geqslant 6 \cdot 3^{m-2}=2 \cdot 3^{m-1} .
$$

2.2. Пусть $\omega^{\sigma} a_{k}=\omega^{\tau} a_{l}, \omega^{\sigma} a_{k} \neq \omega^{\tau+\mu} a_{l}$ и $\omega^{\sigma} a_{k} \neq \omega^{\tau_{\nu}} a_{l}$. Тогда $\sigma=\tau, a_{k}=a_{l}$, откуда следует, что

$$
d\left(\omega^{\sigma} \alpha_{k}, \omega^{\tau} \alpha_{l}\right)=d\left(\omega^{\sigma} a_{k}, \omega^{\sigma+\mu} a_{k}\right)+d\left(\omega^{\sigma} a_{k}, \omega^{\sigma+v} a_{k}\right)=2 \cdot 3^{m-1} .
$$

Аналогично, если $\omega^{\sigma} a_{k}=\omega^{\tau+\mu} a_{l}, \omega^{\sigma} a_{k} \neq \omega^{\tau} a_{l}$ и $\omega^{\sigma} a_{k} \neq \omega^{\tau+v} a_{l}$, то

$$
d\left(\omega^{\sigma} \alpha_{k}, \omega^{\tau} \alpha_{l}\right)=d\left(\omega^{\tau+\mu} a_{l}, \omega^{\tau} a_{l}\right)+d\left(\omega^{\tau+\mu} a_{l}, \omega^{\tau+\nu} a_{l}\right)=2 \cdot 3^{m-1}
$$

а если $\omega^{\sigma} a_{k}=\omega^{\tau+v} a_{l}, \omega^{\sigma} a_{k} \neq \omega^{\tau} a_{l}$ и $\omega^{\sigma} a+k \neq \omega^{\tau+\mu} a_{l}$, то

$$
d\left(\omega^{\sigma} \alpha_{k}, \omega^{\tau} \alpha_{l}\right)=d\left(\omega^{\tau+\nu} a_{l}, \omega^{\tau} a_{l}\right)+d\left(\omega^{\tau+\nu} a_{l}, \omega^{\tau+\mu} a_{l}\right)=2 \cdot 3^{m-1} .
$$

2.3. Если $\omega^{\sigma} a_{k}=\omega^{\tau} a_{l}$ и $\omega^{\sigma} a_{k}=\omega^{\tau+\mu} a_{l}$, то $a_{l}=\omega^{\mu} a_{l}$, что невозможно, так как $\mu \neq 0$. Аналогично, если $\omega^{\sigma} a_{k}=\omega^{\tau} a_{l}, \quad \omega^{\sigma} a_{k}=\omega^{\tau+\nu} a_{l}$, или $\omega^{\sigma} a_{k}=\omega^{\tau+\mu} a_{l}$, $\omega^{\sigma} a_{k}=\omega^{\tau+v} a_{l}$, то опять получаем противоречие.

3. Пусть $(\mu, v)=(0,0)$ и $\delta \neq \lambda-$ ненулевой элемент множества $\{0,1,2,3,4\}$. Этот случай аналогичен рассмотренному выше случаю 2 , так что снова справедливо неравенстBO

$$
d\left(\omega^{\sigma} \alpha_{k}, \omega^{\tau} a_{l}\right) \geqslant 2 \cdot 3^{m-1}
$$

4. Пусть $\delta \neq \lambda$ и $\mu \neq v-$ ненулевые элементы множества $\{0,1,2,3,4\}$. Рассмотрим следующие подслучаи.

4.1. Пусть $\omega^{\sigma} a_{k} \neq \omega^{\tau} a_{l}, \omega^{\sigma+\delta} a_{k} \neq \omega^{\tau+\mu} a_{l}$ и $\omega^{\sigma+\lambda} a_{k} \neq \omega^{\tau+\nu} a_{l}$. По предположению индукщии

$$
\begin{aligned}
d\left(\omega^{\sigma} a_{k}, \omega^{\tau} a_{l}\right) & \geqslant 2 \cdot 3^{m-2}, \\
d\left(\omega^{\sigma+\delta} a_{k}, \omega^{\tau+\mu} a_{l}\right) & \geqslant 2 \cdot 3^{m-2}, \\
d\left(\omega^{\sigma+\lambda} a_{k}, \omega^{\tau+\nu} a_{l}\right) & \geqslant 2 \cdot 3^{m-2},
\end{aligned}
$$

откуда

$$
d\left(\omega^{\sigma} \alpha_{k}, \omega^{\tau} \alpha_{l}\right) \geqslant 6 \cdot 3^{m-2}=2 \cdot 3^{m-1}
$$


4.2. Пусть $\omega^{\sigma} a_{k}=\omega^{\tau} a_{l}, \omega^{\sigma+\delta} a_{k}=\omega^{\tau+\mu} a_{l}$ и $\omega^{\sigma+\lambda} a_{k} \neq \omega^{\tau_{\nu}} a_{l}$. Тогда $\sigma=\tau, a_{k}=a_{l}$, и следовательно,

$$
d\left(\omega^{\sigma} \alpha_{k}, \omega^{\tau} \alpha_{l}\right)=d\left(\omega^{\sigma+\delta} a_{k}, \omega^{\sigma+\mu} a_{k}\right)+d\left(\omega^{\sigma+\lambda} a_{k}, \omega^{\sigma+\nu} a_{k}\right)=2 \cdot 3^{m-1} .
$$

Пусть теперь $\omega^{\sigma+\delta} a_{k}=\omega^{\tau+\mu} a_{l}, \omega^{\sigma} a_{k} \neq \omega^{\tau} a_{l}$ и $\omega^{\sigma+\lambda} a_{k} \neq \omega^{\tau+v} a_{l}$. Тогда $\omega^{\sigma} a_{k}=$ $\omega^{\tau+\mu-\delta} a_{l}, \omega^{\sigma+\lambda} a_{k}=\omega^{\tau+\mu-\delta+\lambda} a_{l}$, и следовательно,

$$
d\left(\omega^{\sigma} \alpha_{k}, \omega^{\tau} \alpha_{l}\right)=d\left(\omega^{\tau+\mu-\delta} a_{l}, \omega^{\mu} a_{l}\right)+d\left(\omega^{\tau+\mu-\delta+\lambda} a_{l}, \omega^{\tau+\nu} a_{l}\right)=2 \cdot 3^{m-1} .
$$

Наконец, пусть $\omega^{\sigma+\lambda} a_{k}=\omega^{\tau+v} a_{l}, \omega^{\sigma} a_{k} \neq \omega^{\tau} a_{l}$ и $\omega^{\sigma+\delta} \neq \omega^{\tau+\mu} a_{l} /$ Используя те же аргументы, опять получаем равенство

$$
d\left(\omega^{\sigma} \alpha_{k}, \omega^{\tau} \alpha_{l}\right)=2 \cdot 3^{m-1} .
$$

4.3. Предположим, что $\omega^{\sigma} a_{k}=\omega^{\tau} a_{l}$ и $\omega^{\sigma+\delta} a_{k}=\omega^{\tau+\mu} a_{l}$. Тогда $\sigma=\tau, a_{k}=a_{l}$, и мы получаем, что

$$
\alpha_{k}=\left(\begin{array}{lll}
a_{k} & \omega^{\delta} a_{k} & \omega^{\lambda} a_{k}
\end{array}\right), \quad \alpha_{l}=\left(\begin{array}{ccc}
a_{k} & \omega^{\delta} a_{k} & \omega^{v} a_{k}
\end{array}\right),
$$

что невозможно в силу (3). Аналогично, если $\omega^{\sigma} a_{k}=\omega^{\tau} a_{l}$ и $\omega^{\sigma+\lambda} a_{k}=\omega^{\tau+v} a_{l}$, то $\sigma=\tau, a_{k}=a_{l}$, откуда следует, что $\lambda=\nu$. Таким образом,

$$
\alpha_{k}=\left(\begin{array}{lll}
a_{k} & \omega^{\delta} a_{k} & \omega^{\lambda} a_{k}
\end{array}\right), \quad \alpha_{l}=\left(\begin{array}{lll}
a_{k} & \omega^{\mu} a_{k} & \omega^{\lambda} a_{k}
\end{array}\right),
$$

что также невозможно в силу (3).

Наконец, если $\omega^{\sigma+\delta} a_{k}=\omega^{\tau+\mu} a_{l}$ и $\omega^{\sigma+\lambda} a_{k}=\omega^{\tau+\nu} a_{l}$, то справедливо равенство $\lambda-\delta=\nu-\mu$, из которого следует, что

$$
\alpha_{k}=\left(\begin{array}{lll}
a_{k} & \omega^{\delta} a_{k} & \omega^{\delta+\kappa} a_{k}
\end{array}\right), \quad \alpha_{l}=\left(\begin{array}{lll}
a_{l} & \omega^{\mu} a_{l} & \omega^{\mu+\kappa} a_{l}
\end{array}\right),
$$

где $\kappa=\lambda-\delta=\nu-\mu$. В силу (3) описанный выше вид векторов $\alpha_{k}$ и $\alpha_{l}$ невозможен, что завершает доказательство.

Следствие 1. Нелинейные пятеричные коды $C_{m}^{*}$ и $\tilde{C}_{m}^{*}$ имеют, соответственно, параметры

$$
\left(3^{m}, 5^{m}, 2 \cdot 3^{m-1}\right), \quad\left(3^{m}, 5^{m+1}, 2 \cdot 3^{m-1}\right)
$$

Доказательство. Достаточно определить минимум расстояния $d$ кодов $C_{m}^{*}$ и $\tilde{C}_{m}^{*}$. Ясно, что минимум расстояния Хемминга кода $C_{m}^{*}$ равен минимуму расстояния Хемминга кода $\widetilde{C}^{*}$ и равен минимуму расстояния Хемминга между различными строками матрицы $H_{m}$. Теперь результат следует из теоремы 3.

Наряду с $C_{m}^{*}$ и $\widetilde{C}_{m}^{*}$ можно рассматривать нелинейные коды $C_{m}$ и $\widetilde{C}_{m}$, состоящие соответственно из строк модифицированных матриц Батсона-Адамара $H_{m}$ и $\tilde{H}_{m}$. Коды $C_{n}, \widetilde{C}_{m}$ имеют те же параметры, что и коды $C_{m}^{*}, \widetilde{C}_{m}^{*}$, и процедуры их декодирования также аналогичны.

Теорема 3. Коды $C_{m}$ и $\widetilde{C}_{m}$ имеют параметры

$$
\left(3^{m}, 5^{m}, 2 \cdot 3^{m-1}\right), \quad\left(3^{m}, 5^{m+1}, 2 \cdot 3^{m-1}\right)
$$

и исправляют любые конфигурачии из $t \leqslant\left[\left(2 \cdot 3^{m-1}-1\right) / 2\right]$ очибок. 
Ясно, что если $a_{k}=\left(a_{k, 1}, \ldots, a_{k, 3^{m}}\right)$ и $\bar{a}_{l}^{\tau}=\left(\bar{a}_{l, 1}, \ldots, \bar{a}_{l, 3^{m}}\right)^{\tau}-$ код-векторы соответственно кода $C_{m}$ и кода $C_{m}^{*}$, то

$$
a_{k} \bar{a}_{l}^{\tau}=\sum_{j=1}^{3^{m}} a_{k j} \bar{a}_{l j}=\gamma_{k l},
$$

где $\gamma_{k l}$-соответствующий элемент матрищы $J_{m}$. Здесь и далее верхний индекс $\tau$ означает транспонирование.

\section{4. Алгоритм декодирования}

Для кодов $C_{m}^{*}, \widetilde{C}_{m}^{*}$ и $C_{m}, \widetilde{C}_{m}$ имеются эффективные процедуры декодирования. Алгоритмы декодирования для $C_{m}^{*}, \tilde{C}_{m}^{*}$ и $C_{m}, \tilde{C}_{m}$ совершенно аналогичны, поэтому мы ограничимся описанием алгоритма декодирования только для $C_{m}^{*}$.

Пусть

$$
H_{m}=\left(a_{k l}\right), \quad 1 \leqslant k \leqslant 5^{m}, \quad 1 \leqslant l \leqslant 3^{m},
$$

- модифицированная $5^{m} \times 3^{m}$ матрица Батсона-Адамара с элементами из $C=\langle\omega\rangle$. Пусть

$$
\bar{a}_{k_{0}}^{\tau}=\left(\bar{a}_{k_{0}, 1}, \ldots, \bar{a}_{k_{0}, 3 m}\right)^{\tau} \in C_{m}^{*}
$$

- передаваемый вектор и

$$
\bar{c}_{k}^{\tau}=\left(\bar{c}_{k, 1}, \ldots, \bar{c}_{k, 3^{m}}\right)^{\tau}
$$

- полученный вектор, отличающийся от $\bar{a}_{k}^{\tau}$ в $t$ позищиях. Предположим, что канал с шумом преобразует каждый символ $\bar{a}_{k l}$ из алфавита $C=\left\{1, \omega, \omega^{2}, \omega^{3}, \omega^{4}\right\}$ в некоторый другой символ из $C$ с одной и той же малой вероятностью $p^{*}$ и не изменяет $\bar{a}_{k l}$ с вероятностью $1-p^{*}$.

Для восстановления передаваемого вектора $\bar{a}_{k_{0}}^{\tau}$ из полученного вектора $\bar{c}^{\tau}$ вычтем $\bar{c}^{\tau}$ из каждого столбца $\bar{a}_{k}^{\tau}$ матрицы $H_{m}^{*}$ и затем рассмотрим полученную в результате этой операции матрицу

$$
S_{m}^{*}=\left(\bar{a}_{k l}-\bar{c}_{l}\right)^{\tau}, \quad 1 \leqslant k \leqslant 5^{m}, \quad 1 \leqslant l \leqslant 3^{m} .
$$

Элементы матрицы $H_{m}^{*}$ и компоненты вектора $\bar{c}^{-\tau}$ являются элементами циклической группы $C=\langle\omega\rangle$, поэтому полученная матрица $S_{m}^{*}=\left(\bar{s}_{k l}\right)^{\tau}-$ это $3^{m} \times 5^{m}$ матрица с элементами

$$
\bar{s}_{k l}=s_{k l}^{(0)}+s_{k l}^{(1)} \omega+s_{k l}^{(2)} \omega^{2}+s_{k l}^{(3)} \omega^{3}
$$

из кольца $\mathbf{Z}[\omega]$.

Рассмотрим множество столбцов

$$
\bar{s}_{k}^{\tau}=\left(\bar{s}_{k, 1}, \ldots, \bar{s}_{k, 3^{m}}\right)^{\tau}=\left(\bar{a}_{k, 1}-\bar{c}_{1}, \ldots, \bar{a}_{k, 3^{m}}-\bar{c}_{3^{m}}\right)^{\tau}
$$

матрицы $S_{m}^{*}$. Если число $t$ искаженных компонент полученного вектора $\bar{c}^{\tau}$ удовлетворяет неравенству

$$
t \leqslant[(d-1) / 2]=\left[\left(2 \cdot 3^{m-1}-1\right) / 2\right],
$$

то расстояние Хемминга $d\left(\bar{a}_{k_{0}}, \bar{c}\right)$ между $\bar{a}_{k_{0}}=\left(\bar{a}_{k_{0}, 1}, \ldots, \bar{a}_{k_{0}, 3^{m}}\right)$ и $\bar{c}=\left(\bar{c}_{1}, \ldots, \bar{c}_{3^{m}}\right)$ равно $t$. Отсюда следует, что столбец

$$
\bar{s}_{k_{0}}^{\tau}=\bar{a}_{k_{0}}^{\tau}-\bar{c}^{\tau}=\left(\bar{s}_{k_{0}, 1}, \ldots, \bar{s}_{k_{0}, 3 m}\right)^{\tau}
$$


матрищы $S_{m}^{*}$ содержит ровно $t$ ненулевых элементов. С другой стороны, если $k \neq k_{0}$, то

$$
2 \cdot 3^{m-1} \leqslant d\left(\bar{a}_{k}, \bar{a}_{k_{0}}\right) \leqslant d\left(\bar{a}_{k}, \bar{c}+d\left(\bar{c}, \bar{a}_{k_{0}}\right)=d\left(\bar{a}_{k}, \bar{c}\right)+t\right.
$$

откуда

$$
d\left(\bar{a}_{k}, \bar{c}\right) \geqslant 2 \cdot 3^{m-1}-t>t .
$$

Таким образом, любой столбец $\bar{s}_{k}^{\tau}$ матрищы $S_{m}^{*}$ с $k \neq k_{0}$ содержит по меньшей мере $t+1$ ненулевой элемент. Это свойство матрищы $S_{m}^{*}$ приводит к следующему простому алгоритму декодирования.

Чтобы восстановить переданный вектор $\bar{a}_{k_{0}}^{\tau}$, проверяем множество столбцов матрицы $S_{m}^{*}$ и выбираем единственный столбец

$$
\bar{s}_{k_{0}}=\left(\bar{s}_{k_{0}, 1}, \ldots, \bar{s}_{k_{0}, 3^{m}}\right)^{\tau}
$$

с минимальным числом $t$ ненулевых элементов.

Затем декодируем полученный вектор $\bar{c}^{\tau}$ соответствующим столбцом

$$
\bar{a}_{k_{0}}^{\tau}=\left(\bar{a}_{k_{0}, 1}, \ldots, \bar{a}_{k_{0}, 3^{m}}\right)^{\tau}
$$

матрицы $H_{m}^{*}$. Ясно, что код $C_{m}^{*}$ исправляет любые конфигурации из

$$
t \leqslant[(d-1) / 2]=\left[\left(2 \cdot 3^{m-1}-1\right) / 2\right]
$$

ошибок.

Аналогично, код $\widetilde{C}_{m}^{*}$ с параметрами $\left(3^{m}, 5^{m+1}, 2 \cdot 3^{m-1}\right)$ также исправляет любые конфигурации из

$$
t \leqslant\left[\left(2 \cdot 3^{m-1}-1\right) / 2\right]
$$

ошибок. В этом случае матрища

$$
S_{m}^{*}=\left(\bar{s}_{k l}\right)^{\tau}=\left(\bar{a}_{k l}-\bar{c}_{l}\right)^{\tau}, \quad 1 \leqslant k \leqslant 5^{m}, \quad 1 \leqslant l \leqslant 3^{m},
$$

- комплексная $3^{m} \times 5^{m+1}$ матрица с элементами из кольца $\mathbf{Z}[\omega]$.

Так как в предложенном алгоритме декодирования фактически используется только операция вычитания алгебраических целых чисел кольца $Z[\omega]$ и не требуется выполнения умножения или деления, этот алгоритм является достаточно быстрым.

\section{5. Пример}

В этом разделе мы проиллюстрируем описанный в предыдущем разделе алгоритм декодирования простейшим примером. Пусть

$$
H_{1}=\left(\begin{array}{ccc}
1 & 1 & 1 \\
1 & \omega & \omega^{2} \\
1 & \omega^{2} & \omega^{4} \\
1 & \omega^{3} & \omega \\
1 & \omega^{4} & \omega^{3}
\end{array}\right), \quad H_{1}^{*}=\left(\begin{array}{ccccc}
1 & 1 & 1 & 1 & 1 \\
1 & \omega^{4} & \omega^{3} & \omega^{2} & \omega \\
1 & \omega^{3} & \omega & \omega^{4} & \omega^{2}
\end{array}\right)
$$


Рассмотрим матрицу

и перепишем ее в вида

$$
H_{2}=\left(\begin{array}{ccc}
H_{1} & H_{1} & H_{1} \\
H_{1} & \omega H_{1} & \omega^{2} H_{1} \\
H_{1} & \omega^{2} H_{1} & \omega^{4} H_{1} \\
H_{1} & \omega^{3} H_{1} & \omega H_{1} \\
H_{1} & \omega^{4} H_{1} & \omega^{3} H_{1}
\end{array}\right)
$$

$$
H_{2}=\left(\begin{array}{ccccccccc}
1 & 1 & 1 & 1 & 1 & 1 & 1 & 1 & 1 \\
1 & \omega & \omega^{2} & 1 & \omega & \omega^{2} & 1 & \omega & \omega^{2} \\
1 & \omega^{2} & \omega^{4} & 1 & \omega^{2} & \omega^{4} & 1 & \omega^{2} & \omega^{4} \\
1 & \omega^{3} & \omega & 1 & \omega^{3} & \omega & 1 & \omega^{3} & \omega \\
1 & \omega^{4} & \omega^{3} & 1 & \omega^{4} & \omega^{3} & 1 & \omega^{4} & \omega^{3} \\
1 & 1 & 1 & \omega & \omega & \omega & \omega^{2} & \omega^{2} & \omega^{2} \\
\vdots & \vdots & \vdots & \vdots & \vdots & \vdots & \vdots & \vdots & \vdots \\
1 & 1 & 1 & \omega^{4} & \omega^{4} & \omega^{4} & \omega^{3} & \omega^{3} & \omega^{3} \\
1 & \omega & \omega^{2} & \omega^{4} & 1 & \omega & \omega^{3} & \omega^{4} & 1 \\
1 & \omega^{2} & \omega^{4} & \omega^{4} & \omega & \omega^{3} & \omega^{3} & \omega & \omega^{4} \\
1 & \omega^{3} & \omega & \omega^{4} & \omega^{2} & 1 & \omega^{3} & \omega^{4} & \omega^{3} \\
1 & \omega^{4} & \omega^{3} & \omega^{4} & \omega^{2} & \omega^{2} & \omega^{3} & \omega^{2} & \omega
\end{array}\right) .
$$

Код $C_{2}^{*}$ состоит из столбцов $3^{2} \times 5^{2}$ матрицы $H_{2}^{*}$ и имеет параметры $\left(3^{2}, 5^{2}, 2 \cdot 3\right)$. Покажем, что код $C_{2}^{*}$ исправляет одну и две ошибки. Пусть код-вектор $\bar{a}^{\tau} \in C_{2}^{*}$ имеет вид

$$
\bar{a}^{\tau}=\left(1, \omega^{3}, \omega, 1, \omega^{3}, \omega, 1, \omega^{3}, \omega\right)^{\tau}
$$

и предположим, что этот вектор передается по каналу с шумом. Пусть

$$
\bar{c}^{\tau}=\left(1, \omega^{3}, \omega, 1, \omega^{3}, \omega, 1,1, \omega^{2}\right)^{\tau}
$$

- полученный вектор, отличающийся от $\bar{a}^{\tau}$ в последних двух позициях. Чтобы исправить ошибки, мы вычтем $\bar{c}^{\tau}$ из каждого столбца матрицы $H_{2}^{*}$. В результате получим матрицу

$$
S_{2}^{*}=\left(\begin{array}{ccccccc}
0 & 0 & 0 & 0 & \ldots & 0 & 0 \\
1-\omega^{3} & \omega^{4}-\omega^{3} & 0 & \omega^{2}-\omega^{3} & \ldots & \omega^{2}-\omega^{3} & \omega-\omega^{3} \\
1-\omega & \omega^{3}-\omega & 0 & \omega^{4}-\omega & \ldots & \omega^{4}-\omega & \omega^{2}-\omega \\
0 & 0 & 0 & 0 & \ldots & \omega-1 & \omega-1 \\
1-\omega^{3} & \omega^{4}-\omega^{3} & 0 & \omega-\omega^{3} & \ldots & 1-\omega & \omega^{3}-\omega \\
0 & 0 & 0 & 0 & \ldots & \omega^{2}-1 & \omega^{2}-1 \\
0 & \omega^{4}-1 & \omega-1 & \omega-1 & \ldots & \omega^{4}-1 & \omega^{3}-1 \\
1-\omega^{2} & \omega^{3}-\omega^{2} & \omega-\omega^{2} & 0 & \ldots & \omega-\omega^{2} & \omega^{4}-\omega^{2}
\end{array}\right)
$$

с элементами из $\mathbf{Z}(\omega)$. Третий столбец этой матрицы содержит только два ненулевых элемента и каждый другой столбец матрицы $S_{2}^{*}$ содержит по менњшй мере три ненулевых элемента. Согласно описанному в предыдущем разделе алгоритму декодирования в качестве декодированного вектора $\bar{c}^{\tau}$ выбираем третий столбец

$$
\bar{a}_{3}^{\tau}=\left(1, \omega^{3}, \omega, 1, \omega^{3}, \omega, 1, \omega^{3}, \omega\right)^{\tau}
$$

матрищы $H_{2}^{*}$. 


\section{Список литературы}

1. Baliga A., New self-dual codes from cocyclic Hadamard matrices. J. Comb. Math. Comb. Comput. (1982) 28, 7-14.

2. Baliga A., Horadam K. J., Cocyclic Hadamard matrices over $\mathbf{Z}_{t} \times \mathbf{Z}_{2}^{2}$. Australian J. Comb. (1995) 11, 123-134.

3. Butson K. T., Generalized Hadamard matrices. Proc. Amer. Math. Soc. (1962) 13, 894-898.

4. Elliot D. F., Rao K. R., Fast transforms. Algorithms, analyses, applications. Academic Press, New York, 1982.

5. Horadam K. J., Udaya P., Cocyclic Hadamard codes. IEEE Trans. Inform. Theory (2000) 46, 15451550 .

6. Maslen D. K., Rockmore D. N., Generalized FFTs-a survey of some recent results. DIMACS Ser. Discr. Math. Theoret. Comp. Sci. (1997) 28, 183-237.

7. Pinnawala N., Rao A., Cocyclic simplex codes of type $\alpha$ over $\mathbf{Z}_{4}$ and $\mathbf{Z}_{2}$. IEEE Trans. Inform. Theory (2004) 50, №9, 2165-2169.

Статья поступила 29.05.2005. 